\title{
Bandwidth and power allocation for cooperative relay in cognitive radio networks
}

\author{
Huogen Yu*, Wanbin Tang and Shaoqian Li
}

\begin{abstract}
In this article, we consider a cognitive radio (CR) relay network where one source secondary user (SU) communicates with its corresponding destination SU with the help of relay SUs. Conventionally, equal bandwidth and/or power are allocated to each relay $S U$, which may not be efficient for the $C R$ with limited bandwidth and power. Therefore, this article presents bandwidth and power allocation with amplify-and-forward (AF) or decode-and-forward (DF) relaying protocol to (1) maximize the sum network throughput; (2) minimize the total transmit power of the CR network with considering the fairness of power drain of relay SUs; (3) maximize the energy efficiency of the CR network. It is shown that DF relaying protocol can achieve better performance when the decoding rate constraint is not considered. In contrast, when considering the decoding rate constraint in DF relaying protocol, we propose the hybrid relaying protocol that combines AF and DF relaying protocols. We formulate the joint bandwidth and power allocation problem with hybrid relaying protocol to maximize the sum network throughput. A greedy algorithm is developed to solve the joint optimization problem, which has much less computational complexity. It is shown that the greedy algorithm has comparable performance to the exhaustive search algorithm. Finally, numerical results are provided to endorse our proposed algorithms.
\end{abstract}

Keywords: Cognitive radio, Relay network, Bandwidth and power allocation, Relaying protocol, Greedy algorithm

\section{Introduction}

Cognitive radio (CR) has emerged as a promising technology to meet the challenge of the spectrum scarcity for the next generation wireless systems [1,2]. CR allows secondary users (SUs) to access the "spaces" in frequency, time, and/or space domains as long as they do not cause harmful interference to primary users (PUs). Generally, there are mainly two paradigms for the operation of CR: opportunistically spectrum access (OSA) [3,4] and spectrum sharing (SS) [5]. OSA allows SUs to transmit only when a frequency band is detected to be idle, while SS allows SUs to transmit simultaneously with the PU over the same frequency as long as the quality of service of PU is guaranteed. In this article, we focus on the SS paradigm.

In order to improve $C R$ network performance, resource allocation in CR networks has been extensively researched (see e.g., [6-9]). In [6], the optimal power allocation strategies which aim at, respectively, maximizing the ergodic,

*Correspondence: yuhuogen@uestc.edu.cn

National Key Laboratory of Science and Technology on Communications, University of Electronic Science and Technology of China, No. 2006 Xiyuan Avenue, Chengdu, China delay-limited and outage capacities of the SU were studied under different combinations of transmit power constraint and interference power constraint. In [7], the optimal bandwidth and power allocations were derived to maximize the sum ergodic capacity of all the SUs under all possible combinations of transmit power constraint and interference power constraint. In [8], the optimal timesharing and power allocation policy was studied under the peak transmit power constraint and average interference power constraint. In [9], the downlink and uplink subcarriers and power allocations were studied under considering the CR out-of-band emissions and spectrum-sensing errors.

On the other hand, in the case of severe channel conditions in direct links, cooperative relay has been introduced to forward the data from source to destination in CR networks. Recent works [10-12] have studied the resource allocation for cooperative relay in CR networks. In [10], relay selection and power allocation with amplify-and-forward (AF) relaying protocol were investigated in cooperative CR systems. In [11], the power and

\section{量 Springer}

(C) 2012 Yu et al; licensee Springer. This is an Open Access article distributed under the terms of the Creative Commons Attribution License (http://creativecommons.org/licenses/by/2.0), which permits unrestricted use, distribution, and reproduction in any medium, provided the original work is properly cited. 
channel allocation with decode-and-forward (DF) relaying protocol was proposed to maximize the overall endto-end throughput in the cooperative relay CR network. In [12], the relay and power allocation schemes with decodeand-forward (DF) relaying protocol were presented for cooperative relay in the CR network.

However, since the total available bandwidth of $\mathrm{CR}$ networks is limited, it is necessary to study the joint bandwidth and power allocation for cooperative relay in $\mathrm{CR}$ networks. Unfortunately, very few works have considered the joint optimization problem. In this article, we study the joint optimization of bandwidth and power allocation for cooperative relay in CR networks, which is especially efficient for the CR with both limited bandwidth and limited power. Meanwhile, unlike majority of research which has focused only on maximizing the throughput of $C R$ networks, various performance metrics for the joint optimization problem have been considered in this article. For some applications which require high data rate, it is preferable to allocate the resource to maximize the CR network throughput. While when the limited transmit power and the fairness issue are considered for the resource allocation, it is preferable to minimize the total transmit power of the CR network with considering the fairness of power drain of relay SUs. Finally, by taking into consideration the total energy consumption of all SUs, energy efficiency is also used as the performance metric. Similar to $[13,14]$, energy efficiency is defined as the number of bits transmitted per unit of energy consumption in this article.

The contributions of this article can be summarized as follows. First, the problems of the joint bandwidth and power allocation with AF or DF relaying protocol are, respectively, formulated to: (1) maximize the sum throughput of the CR network; (2) minimize the total transmit power of the $\mathrm{CR}$ network with considering the fairness of power drain of relay SUs; (3) maximize the energy efficiency of the CR network. We derive the optimal bandwidth allocation with AF relaying protocol to maximize the sum throughput of the CR network for any given power allocation, which results in equivalent problem that only involves power allocation. Second, the corresponding problems which maximize the sum throughput of the CR network and minimize the total transmit power of all SUs can be formulated as optimization problems which are shown to be convex. Therefore, we can solve these problems by using convex optimization techniques. Third, the design of joint bandwidth and power allocation to maximize the energy efficiency of the CR network has been formulated as the fractional programming (FP) problem [15]. To solve it, we transform it to the parametric formulation, which allows convex optimization technology to be applied to find the optimal bandwidth and power allocation strategy. Finally, considering the decoding rate constraint in DF relaying protocol, we propose the hybrid relaying protocol that combines AF and DF relaying protocols. The joint bandwidth and power allocation problem with hybrid relaying protocol is formulated to maximize the sum throughput of the $C R$ network. Moreover, an exhaustive search algorithm and a greedy algorithm are proposed to obtain the optimal solution.

The rest of this article is organized as follows. In Section System model, the system model is given. In Section Joint bandwidth and power allocation with AF or DF relaying protocol, joint bandwidth and power allocations with AF or DF relaying protocol are, respectively, formulated and studied for three performance metrics. In Section Joint bandwidth and power allocation with hybrid relaying protocol, joint bandwidth and power allocation with the hybrid relaying protocol is formulated. Moreover, two optimization algorithms are proposed. In Section Numerical results and discussions, numerical results and discussions are given. Finally, Section Conclusion makes some concluding remarks.

\section{System model}

Consider a CR network with one source SU, one destination $\mathrm{SU}$, and $K$ relay SUs that are employed for forwarding data from source SU to destination SU. It is assumed that the direct link between source SU and destination SU does not exist due to the severe channel condition. Further, it is considered that the SUs are allowed to use the spectrum of bandwidth $W$ which originally assigned to one PU, as long as the quality of service of PU is guaranteed. Here, the spectrum is assumed to undergo flat fading and can be divided into distinct and nonoverlapping channels with unequal bandwidths, so that the SUs share the spectrum through frequency division to avoid interferences with each other. Let $h_{k}^{\mathrm{SR}}$ and $h_{k}^{\mathrm{RD}}$ denote the instantaneous channel gains between source $\mathrm{SU}$ and relay $\mathrm{SU} k$, and between relay SU $k$ and destination SU. Let $g_{\mathrm{SP}}$ and $g_{k}^{\mathrm{RP}}$ denote the instantaneous channel gains between source $\mathrm{SU}$ and PU, and between relay SU $k$ and PU. In this article, we assume that the SUs have a perfect knowledge of channel state information, and this can be realized by estimation and feedback [16].

Of course, in practical systems the bandwidth cannot be divided randomly, and only discrete bandwidth partitioning is possible. For example, in an orthogonal frequency division multiplex (OFDM) system, the bandwidth allocated to each user is specified by an integer number of subcarriers. However, similar to [17], we assume that subcarrier spacing is small enough so that the bandwidth variables can be approximated as to be continuous in this article. Moreover, motivated by the continuous solution, the optimal solution of the discrete case can be achieved through quantizing the optimal solution of the continuous 
case [18]. It is beyond the scope of this article to elaborate more on the topic.

Concerning the relaying procedure, two half-duplex relaying protocols are considered in this article.

1) AF relaying protocol: The relay $\mathrm{SU}$ receives and amplifies the transmitted data from source SU in the first phase, and retransmits the data to destination SU in the second phase. Then, two-hop source-destination link throughput for the $k$ th relaying link with AF relaying protocol can be written as [19]

$$
R_{k, \mathrm{SD}}^{\mathrm{AF}}=W_{k} \log _{2}\left(1+\frac{\frac{P_{k}^{S}\left|h_{k}^{\mathrm{SR}}\right|^{2}}{W_{k} N_{0}} \frac{P_{k}^{R}\left|h_{k}^{\mathrm{RD}}\right|^{2}}{W_{k} N_{0}}}{\frac{P_{k}^{S}\left|h_{k}^{\mathrm{SR}}\right|^{2}}{W_{k} N_{0}}+\frac{P_{k}^{R}\left|h_{k}^{\mathrm{RD}}\right|^{2}}{W_{k} N_{0}}+1}\right),
$$

where $W_{k}$ denotes the channel bandwidth allocated to the $k$ th relaying link. $P_{k}^{S}$ denotes the transmit power of source SU allocated to the $k$ th relaying link. $P_{k}^{R}$ denotes the transmit power of relay SU $k . N_{0}$ stands for the power spectral density (PSD) of additive white Gaussian noise (AWGN).

2) DF relaying protocol: The relay $S U$ receives and decodes the transmitted data from source SU in the first phase, and re-encodes and forwards the data to destination SU in the second phase. Then, the one-hop sourcerelay link throughput for the $k$ th relaying link can be written as

$$
R_{k, \mathrm{SR}}^{\mathrm{DF}}=W_{k} \log _{2}\left(1+\frac{P_{k}^{S}\left|h_{k}^{\mathrm{SR}}\right|^{2}}{W_{k} N_{0}}\right),
$$

and the one-hop relay-destination link throughput for the $k$ th relaying link can be written as

$$
R_{k, \mathrm{RD}}^{\mathrm{DF}}=W_{k} \log _{2}\left(1+\frac{P_{k}^{R}\left|h_{k}^{\mathrm{RD}}\right|^{2}}{W_{k} N_{0}}\right) .
$$

Therefore, two-hop source-destination link throughput for the $k$ th relaying link with DF relaying protocol can be written as

$$
R_{k, \mathrm{SD}}^{\mathrm{DF}}=\min \left\{R_{k, \mathrm{SR}}^{\mathrm{DF}}, R_{k, \mathrm{RD}}^{\mathrm{DF}}\right\}
$$

\section{Joint bandwidth and power allocation with AF or DF relaying protocol}

In this section, the problems of joint bandwidth and power allocation with AF or DF relaying protocol are formulated and solved for different performance metrics. For convenience, we set $\gamma_{0}=\frac{1}{N_{0}}$ in the subsequent discussions, and focus on the high regime of $\gamma_{0}$ in AF relaying protocol. Therefore, the two-hop source-destination link throughput for the $k$ th relaying link with AF relaying protocol can be approximated as

$$
R_{k, \mathrm{SD}}^{\mathrm{AF}} \sim W_{k} \log _{2}\left(1+\frac{P_{k}^{S} P_{k}^{R}\left|h_{k}^{\mathrm{SR}}\right|^{2}\left|h_{k}^{\mathrm{RD}}\right|^{2} \gamma_{0}}{P_{k}^{S}\left|h_{k}^{\mathrm{SR}}\right|^{2} W_{k}+P_{k}^{R}\left|h_{k}^{\mathrm{RD}}\right|^{2} W_{k}}\right) .
$$

Moreover, in this section, we assume that relay SU will be able to execute DF relaying protocol if the data rate between source $\mathrm{SU}$ and relay $\mathrm{SU}$ is greater than zero. In other words, there is no decoding rate constraint in DF relaying protocol.

\section{Sum throughput maximization}

1) AF relaying protocol

For AF relaying protocol, the joint bandwidth and power allocation problem aiming at maximizing the sum throughput of the CR network can be formulated as follows:

Problem P1:

$$
\begin{array}{ll}
\max _{W_{k}, P_{k}^{S}, P_{k}^{R}} & \sum_{k=1}^{K} R_{k, \mathrm{SD}}^{\mathrm{AF}} \\
\text { s.t. } & \sum_{k=1}^{K} W_{k} \leq W \\
& \sum_{k=1}^{K}\left(P_{k}^{S}+P_{k}^{R}\right) \leq P_{\text {th }} \\
& \sum_{k=1}^{K}\left|g_{\mathrm{SP}}\right|^{2} P_{k}^{S} \leq I_{\text {th }} \\
& \sum_{k=1}^{K}\left|g_{k}^{\mathrm{RP}}\right|^{2} P_{k}^{R} \leq I_{\text {th }}
\end{array}
$$

where $P_{\text {th }}$ is the maximum total power that can be used for transmission. $I_{\text {th }}$ is the maximum allowed interference to PU band.

Proposition 1. For any given power $P_{k}^{S}$ and $P_{k}^{R}(k=$ $1,2, \ldots, K)$, the optimal bandwidth allocation of Problem P1 can be found as

$$
W_{k}^{*}=W \frac{\frac{P_{k}^{S} P_{k}^{R}\left|h_{k}^{\mathrm{SR}}\right|^{2}\left|h_{k}^{\mathrm{RD}}\right|^{2} \gamma_{0}}{P_{k}^{S}\left|h_{k}^{\mathrm{SR}}\right|^{2}+P_{k}^{R}\left|h_{k}^{\mathrm{RD}}\right|^{2}}}{\sum_{k=1}^{K} \frac{P_{k}^{S} P_{k}^{R}\left|h_{k}^{\mathrm{SR}}\right|^{2}\left|h_{k}^{\mathrm{RD}}\right|^{2} \gamma_{0}}{P_{k}^{S}\left|h_{k}^{\mathrm{SR}}\right|^{2}+P_{k}^{R}\left|h_{k}^{\mathrm{RD}}\right|^{2}}} .
$$

Proof. See Appendix 1.

According to Proposition 1, Problem P1 can be equivalently written as

Problem P2:

$$
\begin{array}{ll}
\max _{P_{k}^{S}, P_{k}^{R}} & W \log _{2}\left(1+\frac{1}{W} \sum_{k=1}^{K} \frac{P_{k}^{S} P_{k}^{R}\left|h_{k}^{\mathrm{SR}}\right|^{2}\left|h_{k}^{\mathrm{RD}}\right|^{2} \gamma_{0}}{P_{k}^{S}\left|h_{k}^{\mathrm{SR}}\right|^{2}+P_{k}^{R}\left|h_{k}^{\mathrm{RD}}\right|^{2}}\right) \\
\text { s.t. } \quad(8)(9)(10)
\end{array}
$$


Furthermore, Problem P2 is equivalent to Problem P3:

$$
\begin{array}{ll}
\max _{P_{k}^{S}, P_{k}^{R}} & \sum_{k=1}^{K} \frac{P_{k}^{S} P_{k}^{R}\left|h_{k}^{\mathrm{SR}}\right|^{2}\left|h_{k}^{\mathrm{RD}}\right|^{2} \gamma_{0}}{P_{k}^{S}\left|h_{k}^{\mathrm{SR}}\right|^{2}+P_{k}^{R}\left|h_{k}^{\mathrm{RD}}\right|^{2}} \\
\text { s.t. } & (8)(9)(10)
\end{array}
$$

Proposition 2. The objective function of Problem P3 is concave in $P_{k}^{S}$ and $P_{k}^{R}(k=1,2, \ldots, K)$.

\section{Proof. See Appendix 2.}

Obviously, the constraint conditions of Problem P3 are convex. Therefore, Problem P3 is a convex optimization problem. Some standard numerical algorithms for convex optimization can be used to find the optimal solution.

2) DF relaying protocol

For DF relaying protocol, the joint bandwidth and power allocation problem aiming at maximizing the sum throughput of the CR network can be formulated as follows:

Problem P4:

$$
\begin{array}{cl}
\max _{W_{k}, P_{k}^{S}, P_{k}^{R}} & \sum_{k=1}^{K} R_{k, \mathrm{SD}}^{\mathrm{DF}} \\
\text { s.t. } & (7)(8)(9)(10)
\end{array}
$$

Similar to [20], through introducing new variables $T_{k}$, Problem P4 can be equivalently written as

Problem P5:

$$
\begin{array}{cl}
\max _{W_{k}, P_{k}^{S}, P_{k}^{R}, T_{k}} & \sum_{k=1}^{K} T_{k} \\
\text { s.t. } & T_{k}-R_{k, \mathrm{SR}}^{\mathrm{DF}} \leq 0, \quad T_{k}-R_{k, \mathrm{RD}}^{\mathrm{DF}} \leq 0, \\
& k=1,2, \ldots, K \\
& (7)(8)(9)(10)
\end{array}
$$

It is obvious that $R_{k, \mathrm{SR}}^{\mathrm{DF}}$ and $R_{k, \mathrm{RD}}^{\mathrm{DF}}$ are joint concave functions of $W_{k}, P_{k}^{S}$, and $P_{k}^{R}$. Therefore, Problem P5 is a convex optimization problem. According to the well known relationship on the harmonic mean

$$
\frac{\frac{P_{k}^{S}\left|h_{k}^{\mathrm{SR}}\right|^{2}}{W_{k} N_{0}} \frac{P_{k}^{R}\left|h_{k}^{\mathrm{RD}}\right|^{2}}{W_{k} N_{0}}}{\frac{P_{k}^{S}\left|h_{k}^{\mathrm{SR}}\right|^{2}}{W_{k} N_{0}}+\frac{P_{k}^{R}\left|h_{k}^{\mathrm{RD}}\right|^{2}}{W_{k} N_{0}}} \leq \min \left\{\frac{P_{k}^{S}\left|h_{k}^{\mathrm{SR}}\right|^{2}}{W_{k} N_{0}}, \frac{P_{k}^{S}\left|h_{k}^{\mathrm{SR}}\right|^{2}}{W_{k} N_{0}}\right\}
$$

we can easy to show that the sum throughput in DF relaying protocol is superior to that in AF relaying protocol. This conclusion is also validated by latter numerical simulation results.

\section{Power minimization with considering the fairness}

In the problem of maximizing sum throughput, the fairness of relay SUs is not considered. In general, fairness could be defined in terms of different parameters of the system [21]. In this article, we focus on the fairness of power drain of relay SUs. When the differences of relay SUs' channel power gains are large, it is possible that relay SUs with higher channel power gains will consume most of power. Relay SUs with lower channel power gains might not need to consume any power. This will result in a lower survival time of some relay SUs and the CR network. Moreover, by taking into consideration the limited transmit power of the CR network, we minimize the total transmit power of the CR network simultaneously.

1) AF relaying protocol

For AF relaying protocol, the joint bandwidth and power allocation problem aiming at minimize the total transmit power of the CR network can be formulated as follows:

Problem P6:

$$
\begin{array}{cl}
\min _{W_{k}, P_{k}^{S}, P_{k}^{R}} & \sum_{k=1}^{K}\left(P_{k}^{S}+P_{k}^{R}\right) \\
\text { s.t. } & r_{k}-R_{k, \mathrm{SD}}^{\mathrm{AF}} \leq 0, \quad k=1,2, \ldots, K \\
& P_{1}^{R}: P_{2}^{R}: \cdots: P_{K}^{R}=\alpha_{1}: \alpha_{2}: \cdots: \alpha_{K}
\end{array}
$$$$
\text { (7) (8)(9)(10) }
$$

where $r_{k}$ is the minimum acceptable throughput for $k$ th relaying link. $\left\{\alpha_{1}, \alpha_{2}, \ldots, \alpha_{K}\right\}$ is the set of predetermined proportional constraints that are used to ensure fairness. In this article, we set $\alpha_{1}=\alpha_{2}=\cdots=\alpha_{K}=1$.

Proposition 3. The function $R_{k, \mathrm{SD}}^{\mathrm{AF}}$ of Problem P6 is concave in $W_{k}, P_{k}^{S}$, and $P_{k}^{R}(k=1,2, \ldots, K)$.

Proof. The proof is similar to Proposition 2, and is omitted for brevity in this article.

According to Proposition 3, we can know that Problem P6 is also a convex optimization problem. The optimal solution can be efficiently obtained.

2) DF relaying protocol

For DF relaying protocol, The joint bandwidth and power allocation problem aiming at minimize the total transmit power of the CR network can be formulated as follows:

Problem P7:

$$
\begin{array}{cl}
\min _{W_{k}, P_{k}^{S}, P_{k}^{R}} & \sum_{k=1}^{K}\left(P_{k}^{S}+P_{k}^{R}\right) \\
\text { s.t. } & r_{k}-R_{k, \mathrm{SD}}^{\mathrm{DF}} \leq 0, \quad k=1,2, \ldots, K \\
& P_{1}^{R}: P_{2}^{R}: \cdots: P_{K}^{R}=\alpha_{1}: \alpha_{2}: \cdots: \alpha_{K} \\
& (7)(8)(9)(10)
\end{array}
$$


The solution of Problem P7 is similar to the solution of Problem P6. In order to save space, the description will not be repeated again.

\section{Energy efficient}

1) AF relaying protocol

Energy efficiency in the CR network is another widely considered design objective. Therefore, for AF relaying protocol, the corresponding joint bandwidth and power allocation problem aiming at maximizing energy efficiency can be formulated as follows:

Problem P8:

$$
\begin{aligned}
f=\max _{W_{k}, P_{k}^{S}, P_{k}^{R}} & \frac{\sum_{k=1}^{K} R_{k, \mathrm{SD}}^{\mathrm{AF}}}{\sum_{k=1}^{K}\left(P_{k}^{S}+P_{k}^{R}\right)} \\
\text { s.t. } \quad & r_{k}-R_{k, \mathrm{SD}}^{\mathrm{AF}} \leq 0, \quad k=1,2, \ldots, K \\
& (7)(9)(10)
\end{aligned}
$$

Let $Z$ denotes the set of a solution to Problem P8 and $f_{Z}$ denotes the energy efficiency achieved by using the set $Z$. Thus, the set of the optimal solution is given by $Z^{*}=\arg \max f_{Z}$. The optimization Problem P8 belongs to the FP problem, which is difficult to solve directly. Instead, we can transform the fractional programming to the parametric formulation, which allows convex optimization technology to be applied to find the optimal bandwidth and power allocation strategy. Similar to [14], the parametric formulation can be given as follows:

Problem P9:

$$
\begin{aligned}
g(\lambda)=\max _{W_{k}, P_{k}^{S}, P_{k}^{R}} & \sum_{k=1}^{K} R_{k, \mathrm{SD}}^{\mathrm{AF}}-\lambda \sum_{k=1}^{K}\left(P_{k}^{S}+P_{k}^{R}\right) \\
\text { s.t. } & (7)(9)(10)(31)
\end{aligned}
$$

where $\lambda$ is a given value. Let $Z(\lambda)$ denotes the set of a solution to Problem P9 and $g_{Z(\lambda)}(\lambda)$ denotes the value achieved by using the set $Z(\lambda)$. Thus, the set of the optimal solution for a given value of $\lambda$ is given by $Z^{*}(\lambda)=$ $\arg \max g_{Z(\lambda)}(\lambda)$. According to Proposition 3, we can know $Z(\lambda)$

that Problem P9 is a convex optimization problem for a given value of $\lambda$.

Therefore, the relationship between Problem P8 and Problem P9 is established as follows.

Proposition 4. $f_{Z^{*}}=\lambda^{*}$ if and only if $g_{Z^{*}(\lambda)}\left(\lambda^{*}\right)=0$.

Proof. The proof is similar to Proposition 1 in [14], and is omitted for brevity in this article.

Proposition 5. $g_{Z^{*}(\lambda)}(\lambda)$ is a monotonously decreasing function of $\lambda$.
Proof. The proof is similar to Proposition 2 in [14], and is omitted for brevity in this article.

According to Proposition 4, we can know that if we can find an optimal $\lambda^{*}$ such that the optimal value $g_{Z^{*}(\lambda)}\left(\lambda^{*}\right)$ of Problem P9 is 0, then the corresponding optimal solution of Problem P9 is also optimal for Problem P8. Furthermore, instead of using exhaustive search to identify the optimal $\lambda^{*}$, we can use a more efficient bisection search method according to Proposition 5. Algorithm 1 gives the method for finding the optimal $\lambda^{*}$. In Algorithm 1 , the interval $\left[\lambda_{\min }, \lambda_{\max }\right]$ is selected to contain $\lambda^{*}$, and $\varepsilon$ is a predefined small constant.

\section{Algorithm 1: Find the optimal $\lambda^{*}$.}

1. Given: $\lambda \in\left[\lambda_{\min }, \lambda_{\max }\right]$

\section{Repeat}

a) $\lambda \leftarrow\left(\lambda_{\min }+\lambda_{\max }\right) / 2$

b) Solve convex optimization Problem P9 and get $g_{Z^{*}(\lambda)}(\lambda)$

c) if $g_{Z^{*}(\lambda)}(\lambda) \leq 0$, set $\lambda_{\max } \leftarrow \lambda$

else set $\lambda_{\min } \leftarrow \lambda$

3. Until $\lambda_{\max }-\lambda_{\min } \leq \varepsilon$

2) DF relaying protocol

For DF relaying protocol, the joint bandwidth and power allocation problem aiming at maximizing energy efficiency can be formulated as follows:

Problem P10:

$$
\begin{array}{cl}
\max _{W_{k}, P_{k}^{S}, P_{k}^{R}} & \frac{\sum_{k=1}^{K} R_{k, \mathrm{SD}}^{\mathrm{DF}}}{\sum_{k=1}^{K}\left(P_{k}^{S}+P_{k}^{R}\right)} \\
\text { s.t. } & r_{k}-R_{k, \mathrm{SD}}^{\mathrm{DF}} \leq 0, \quad k=1,2, \ldots, K
\end{array}
$$

$$
\text { (7)(9)(10) }
$$

The solution of Problem P10 is similar to the solution of Problem P8. In order to save space, the description will not be repeated again.

\section{Joint bandwidth and power allocation with hybrid relaying protocol}

In previous section, we assume that relay SU can execute the DF relaying protocol if the data rate between source $\mathrm{SU}$ and relay SU is greater than zero. But actually the data rate between source $\mathrm{SU}$ and relay $\mathrm{SU}$ should be higher than $r(r>0)$ to ensure reliable decoding in relay SU. This decoding rate constraint will cause that some poor quality of relaying link may require more resource. In this case, a part of resource will be wasted. In this section, we propose a hybrid relaying protocol to overcome the problem, in which a relay SU uses the AF relaying protocol 
only if it cannot reliably decode the source data. Otherwise, a relay SU uses the DF relaying protocol. Therefore, the joint bandwidth and power allocation problem with hybrid relaying protocol can be formulated as follows:

Problem P11:

$$
\begin{array}{cl}
\max _{W_{k}, P_{k}^{S}, P_{k}^{R}, t_{k}} & \sum_{k=1}^{K}\left[t_{k} R_{k, \mathrm{SD}}^{\mathrm{DF}}+\left(1-t_{k}\right) R_{k, \mathrm{SD}}^{\mathrm{AF}}\right] \\
\text { s.t. } & t_{k}\left(r-R_{k, \mathrm{SR}}^{\mathrm{DF}}\right) \leq 0, \quad k=1,2, \ldots, K \\
& r_{k}-\left[t_{k} R_{k, \mathrm{SD}}^{\mathrm{DF}}+\left(1-t_{k}\right) R_{k, \mathrm{SD}}^{\mathrm{AF}}\right] \leq 0, \\
& k=1,2, \ldots, K \\
& (7)(8)(9)(10)
\end{array}
$$

where $\boldsymbol{t}=\left[t_{1}, t_{2}, \ldots, t_{K}\right]$ is a binary vector which indicates the transmission mode of the relay SUs. If $t_{k}=1$ (resp. $\left.t_{k}=0\right)$, it denotes the relay SU uses the DF relaying protocol (resp. the AF relaying protocol). $r_{k}$ is the minimum acceptable throughput for $k$ th relaying link. $r$ is the relay SU's decoding rate constraint when the relay SU uses the DF relaying protocol.

In general, it is difficult to find the optimal solution for Problem P11 directly. In the following, we will, respectively, develop the exhaustive search algorithm and the greedy algorithm to obtain the optimal solution.

\section{Exhaustive search algorithm}

Obviously, we can obtain the optimal solution by exhaustive search for $\boldsymbol{t}$. Therefore, for a specific $\boldsymbol{t}$, Problem P11 can be transformed to Problem P12 through introducing new variables $T_{k}$ :

Problem P12 (for a specific $\boldsymbol{t}$ ):

$$
\begin{array}{cl}
\max _{W_{k}, P_{k}^{S}, P_{k}^{R}, T_{k}} & R\left(W_{k}, P_{k}^{S}, P_{k}^{R}, T_{k}\right)=\sum_{k=1}^{K} T_{k} \\
\text { s.t. } & t_{k}\left(r-R_{k, \mathrm{SR}}^{\mathrm{DF}}\right) \leq 0, \quad k=1,2, \ldots, K \\
& r_{k}-\left[t_{k} R_{k, \mathrm{SR}}^{\mathrm{DF}}+\left(1-t_{k}\right) R_{k, \mathrm{SD}}^{\mathrm{AF}}\right] \leq 0, \\
& k=1,2, \ldots, K \\
& r_{k}-\left[t_{k} R_{k, \mathrm{RD}}^{\mathrm{DF}}+\left(1-t_{k}\right) R_{k, \mathrm{SD}}^{\mathrm{AF}}\right] \leq 0, \\
& k=1,2, \ldots, K \\
& T_{k}-\left[t_{k} R_{k, \mathrm{SR}}^{\mathrm{DF}}+\left(1-t_{k}\right) R_{k, \mathrm{SD}}^{\mathrm{AF}}\right] \leq 0, \\
& k=1,2, \ldots, K \\
& T_{k}-\left[t_{k} R_{k, \mathrm{RD}}^{\mathrm{DF}}+\left(1-t_{k}\right) R_{k, \mathrm{SD}}^{\mathrm{AF}}\right] \leq 0, \\
& k=1,2, \ldots, K \\
& (7)(8)(9)(10)
\end{array}
$$

As discussed in Section Joint bandwidth and power allocation with $\mathrm{AF}$ or $\mathrm{DF}$ relaying protocol, $R_{k, \mathrm{SR}}^{\mathrm{DF}}, R_{k, \mathrm{RD}}^{\mathrm{DF}}$, and
$R_{k, \mathrm{SD}}^{\mathrm{AF}}$ are jointly concave functions of $W_{k}, P_{k}^{S}$, and $P_{k}^{R}$, respectively. Therefore, Problem P12 is convex and can be solved efficiently. It is clear that the number of solving the optimization Problem P12 in the exhaustive search algorithm is $2^{K}$. When $K$ is large, the exhaustive search algorithm has a very high complexity.

\section{Greedy algorithm}

The greedy algorithm is described as following.

Greedy algorithm: Find the optimal $t_{k}, W_{k}, P_{k}^{S}$, and $P_{k}^{R}$ for Problem P11.

Step1: Perform the initialization as follows:

$$
\begin{array}{rlrl}
\Omega_{(0)} & =\{1,2, \ldots, K\}, & J_{(0)}=\emptyset \\
\boldsymbol{t}_{(0)} & =[0,0, \ldots, 0], & R_{(0)}=0 \\
n & =1 & &
\end{array}
$$

At the $n$th iteration:

Step2: if $n>K$, go to Step 4. Otherwise

$$
\Omega_{(n)}=\{1,2, \ldots, K\}-J_{(n-1)}
$$

for each relaying link $k \in \Omega_{(n)}$ do

$$
\boldsymbol{t}=\boldsymbol{t}_{(n-1)}, \quad t_{k}=1
$$

Solve the optimization Problem P12 for the specific $\boldsymbol{t}$, and get the sum throughput $R_{(n), k}=$

$R\left(W_{k}, P_{k}^{S}, P_{k}^{R}, T_{k}\right)$ (If the optimization Problem P12 for the specific $\boldsymbol{t}$ has no feasible solution,

the sum throughput $\left.R_{(n), k}=0\right)$.

\section{end for}

Step3: if $\max _{k \in \Omega_{(n)}} R_{(n), k} \leq R_{(n-1)}$, go to Step 4 .

Otherwise

$$
\begin{aligned}
j_{(n)} & =\underset{k \in \Omega_{(n)}}{\arg \max } R_{(n), k}, & J_{(n)}=J_{(n-1)} \cup\left\{j_{(n)}\right\} \\
\boldsymbol{t}_{(n)} & =\boldsymbol{t}_{(n-1)}, & t_{j_{(n)}}=1 \\
R_{(n)} & =R_{(n), j_{(n)}}, & n=n+1
\end{aligned}
$$

Go to Step 2.

Step4: $\boldsymbol{t}_{(n-1)}$ is the optimal $\boldsymbol{t}$. Finally, the optimal $W_{k}, P_{k}^{S}$, and $P_{k}^{R}$ can be got through solving the optimization Problem P12 for the optimal $t$.

At the initial stage of the greedy algorithm, we assume that all relay SUs choose AF transmission mode. In the greedy algorithm, $\Omega_{(n)}$ is termed as the candidate set which contains the indices of relay SUs that have not chosen DF transmission mode and are eligible to choose DF transmission mode at the $n$th iteration. $J_{(n)}$ contains the 
indices of relay SUs that have chosen DF transmission mode after the $n$th iteration. $\boldsymbol{t}_{(n)}$ denotes the transmission mode of all relay SUs after the $n$th iteration. $R_{(n)}$ is the sum throughput achieved on the specific transmission mode $\boldsymbol{t}_{(n)} \cdot j_{(n)}$ is the index of relay SU that chooses DF transmission mode at the $n$th iteration. The basic idea of our proposed greedy algorithm is as follows. At each iteration, all relay SUs that have not chosen DF transmission mode will be selected separately. For each selected relay SU at this iteration, its AF transmission mode will be changed to DF transmission mode, which will form a new $\boldsymbol{t}$. By solving the optimization Problem P12 for the new $\boldsymbol{t}$, the sum throughput is obtained. Finally, the relay SU that gives the largest sum throughput at this iteration changes initial AF transmission mode to DF transmission mode. The greedy algorithm will repeat these procedures for the rest of relay SUs that have not chosen DF transmission mode until all relay SUs have chosen DF transmission mode or the sum throughput stops increasing from one iteration to the next.

It is clear that the number of solving the optimization Problem P12 in the greedy algorithm is at most $\frac{K(K+1)}{2}$. Therefore, the complexity of the greedy algorithm is reduced greatly compared with the exhaustive algorithm when $K$ is large.

\section{Numerical results and discussions}

In this section, numerical results and discussions are presented to demonstrate the effectiveness of our proposed algorithms. We set $\gamma_{0}=30 \mathrm{~dB}$ and $r=1$. We also set $W=1, r_{k}=0.5, \forall k, P_{\text {th }}=0 \mathrm{dBW}$ and $I_{\text {th }}=-20 \mathrm{dBW}$ as default values if no other values are specified. For simplicity, all the channel gains are assumed to follow Rayleigh fading. The variance of $h_{k}^{\mathrm{SR}}$ and $h_{k}^{\mathrm{RD}}$ is set to be $0 \mathrm{~dB}$, and the variance of $g_{\mathrm{SP}}$ and $g_{k}^{\mathrm{RP}}$ is set to be $-10 \mathrm{~dB}$. The numerical results presented in this article are obtained by Monte Carlo simulation.

To better understand the superiority of our proposed optimal joint bandwidth and power allocation (OBPA), we compare it with equal bandwidth with optimal power allocation (EBOPA) in this section.

In Figure 1, maximum sum throughput versus maximum total power $P_{\text {th }}$ is presented for AF and DF relaying protocols under maximum allowed interference $I_{\text {th }}=$ $-20 \mathrm{dBW}$. In Figure 2, maximum sum throughput versus maximum allowed interference $I_{\text {th }}$ is presented for AF and DF relaying protocols under maximum total power $P_{\text {th }}=0 \mathrm{dBW}$. In Figure 3, maximum sum throughput versus bandwidth $W$ is presented for $\mathrm{AF}$ and $\mathrm{DF}$ relaying protocols under $P_{\text {th }}=0 \mathrm{dBW}$ and $I_{\text {th }}=-20 \mathrm{dBW}$. It is clear from these figures that maximum sum throughput achieved in DF relaying protocol is superior to that achieved in AF relaying protocol when using the OBPA or EBOPA scheme. It can be also observed that maximum

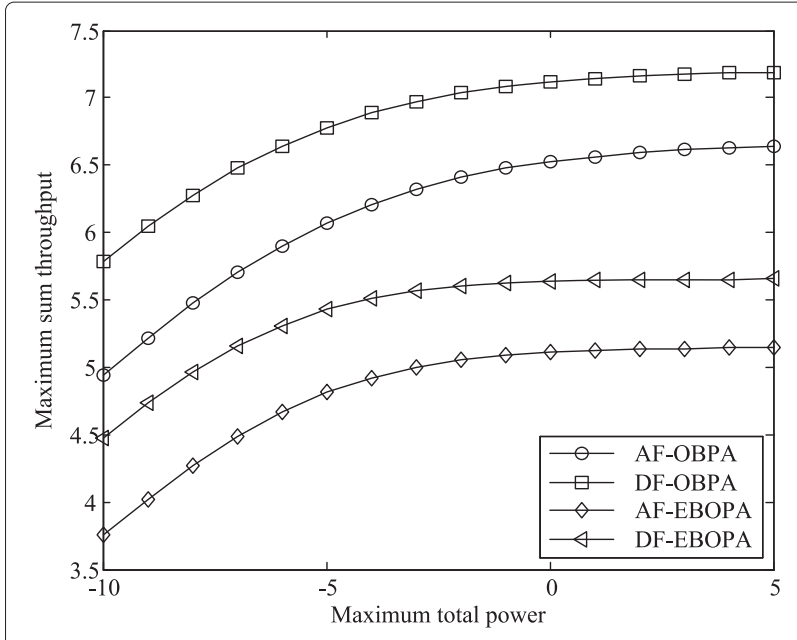

Figure 1 Maximum sum throughput versus maximum total power under $I_{\mathrm{th}}=-20 \mathrm{dBW}$.

sum throughput achieved in the OBPA scheme is larger than that achieved in the EBOPA scheme when using the DF or AF relaying protocol. Furthermore, from Figures 1 and 2 , it can be seen that when $P_{\text {th }}\left(I_{\text {th }}\right)$ reaches a specific value, the maximum sum throughput becomes flat. This indicates that $I_{\text {th }}\left(P_{\text {th }}\right)$ becomes the dominant constraint. From Figure 3, it can be seen that the performance improvement for the OBPA scheme is higher when bandwidth $W$ is larger.

In Figure 4, minimum transmit power of the CR network versus $R\left(R=r_{1}=r_{2}=\cdots=r_{K}\right)$ is presented for AF and DF relaying protocols under $P_{\text {th }}=0 \mathrm{dBW}$ and $I_{\text {th }}=-20 \mathrm{dBW}$. In Figure 5, minimum transmit power of the CR network versus bandwidth $W$ is presented for AF and DF relaying protocols under $P_{\text {th }}=0 \mathrm{dBW}$ and

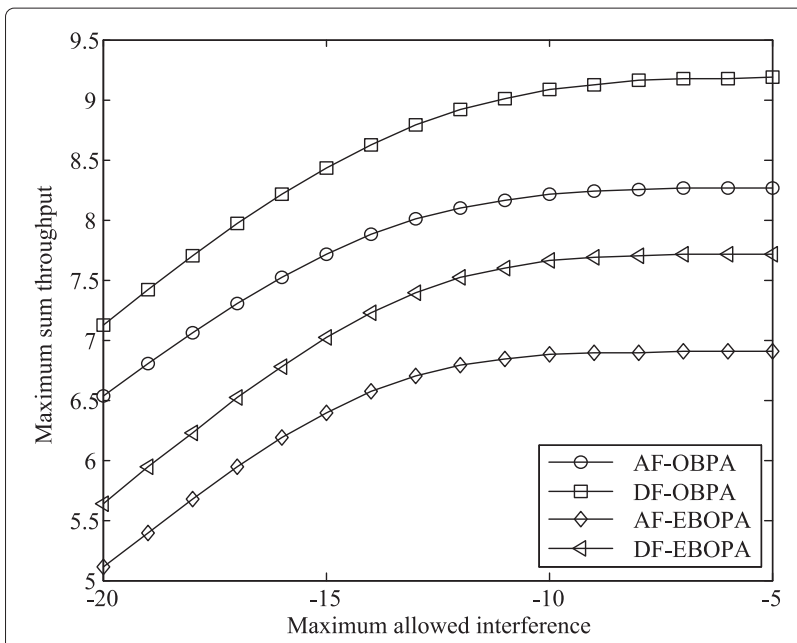

Figure 2 Maximum sum throughput versus maximum allowed interference under $\boldsymbol{P}_{\mathrm{th}}=\mathbf{0} \mathrm{dBW}$. 


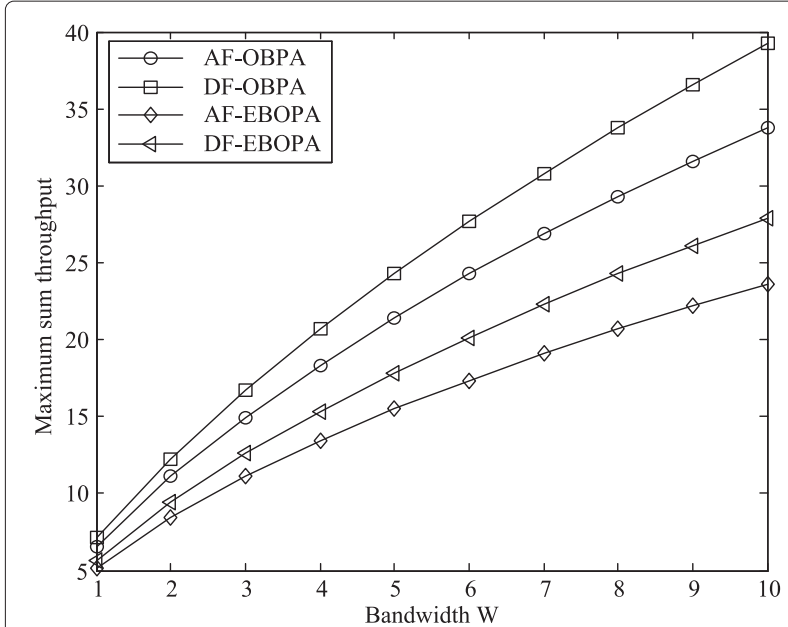

Figure 3 Maximum sum throughput versus bandwidth $W$ under $P_{\text {th }}=0 \mathrm{dBW}$ and $I_{\text {th }}=-20 \mathrm{dBW}$.

$I_{\text {th }}=-20 \mathrm{dBW}$. It is clear from these two figures that minimum transmit power achieved in DF relaying protocol is superior to that achieved in AF relaying protocol when using the OBPA or EBOPA scheme. Moreover, minimum transmit power of the OBPA scheme is always less than that of the EBOPA scheme when using the DF or AF relaying protocol. Furthermore, minimum total transmit power difference between the OBPA scheme and EBOPA scheme is larger when $R$ is larger, or when $W$ is smaller. This indicates that more power is saved through the joint bandwidth and power optimization when the fairness of relay SUs is considered.

In Figure 6, energy efficient versus bandwidth $W$ is presented for $\mathrm{AF}$ and $\mathrm{DF}$ relaying protocols under $P_{\text {th }}=$ $0 \mathrm{dBW}$ and $I_{\text {th }}=-20 \mathrm{dBW}$. It is clear from the figure

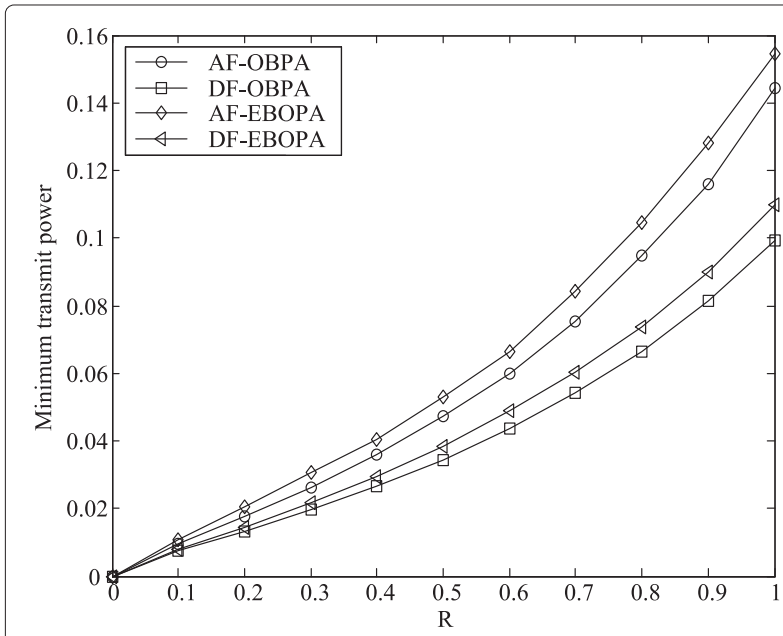

Figure 4 Minimum transmit power of the CR network versus $R$ under $P_{\text {th }}=0 \mathrm{dBW}$ and $I_{\text {th }}=-20 \mathrm{dBW}$.

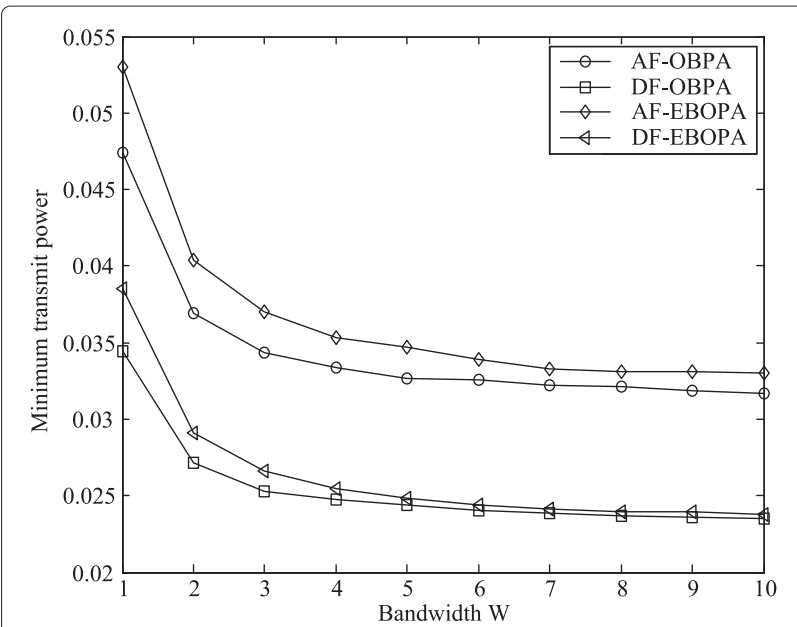

Figure 5 Minimum transmit power of the $C R$ network versus $W$ under $P_{\text {th }}=0 \mathrm{dBW}$ and $I_{\text {th }}=-20 \mathrm{dBW}$.

that energy efficient in DF relaying Protocol is superior to that in AF relaying protocol when using the OBPA or EBOPA scheme. It also can be observed that energy efficient achieved in the OBPA scheme is larger than that achieved in the EBOPA scheme when using the DF or AF relaying protocols. Furthermore, it can be seen that energy efficient increases with the increase of $W$.

In Figure 7, maximum sum throughput with the exhaustive search algorithm and the greedy algorithm versus bandwidth $W$ is presented for hybrid Relaying Protocol under $P_{\text {th }}=0 \mathrm{dBW}$ and $I_{\text {th }}=-20 \mathrm{dBW}$. It is clear from the figure that the greedy algorithm has no performance loss compared to the exhaustive search algorithm, and the maximum sum throughput increases with increasing bandwidth $W$.

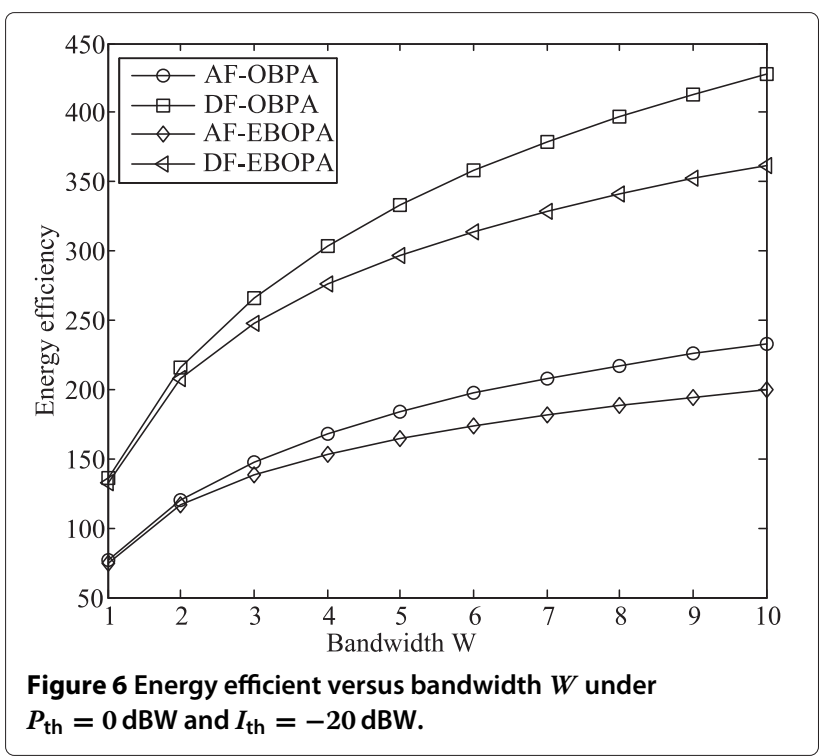




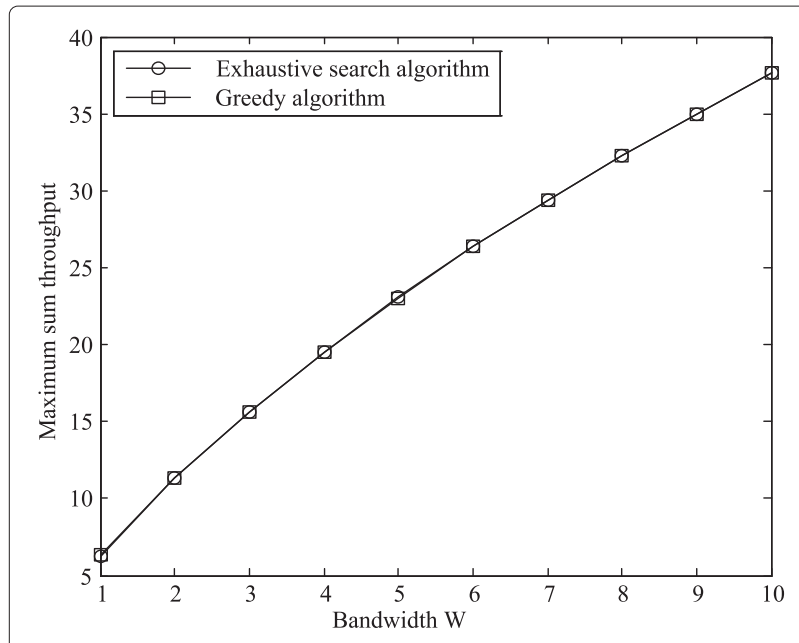

Figure 7 Maximum sum throughput versus bandwidth $W$ for hybrid relaying protocol.

\section{Conclusion}

In this article, we have studied the problems of bandwidth and power allocation in the CR relay network. Particularly, we have presented bandwidth and power allocations with AF or DF relaying protocol to (1) maximize the sum network throughput; (2) minimize the total transmit power of the CR network with considering the fairness of power drain of relay SUs; (3) maximize the energy efficiency of the CR network. It is shown that the corresponding resource allocation problems were equivalently reformulated as convex optimization problems and, therefore, can be solved efficiently. Furthermore, it is shown that DF relaying protocol can achieve better performance when the decoding rate constraint is not considered. In contrast, when considering the decoding rate constraint in DF relaying protocol, we have further proposed the hybrid relaying protocol, which combines $\mathrm{AF}$ and DF relaying protocols. The joint bandwidth and power allocation problem with hybrid relaying protocol was formulated to maximize the sum network throughput. In order to reduce computational complexity of this problem, the greedy algorithm which has comparable performance to the exhaustive search algorithm has been developed to obtain the optimal solution. Finally, the performance of our algorithms has been evaluated through numerical results.

\section{Appendix 1}

For given power $P_{k}^{S}$ and $P_{k}^{R}(k=1,2, \ldots, K)$, Problem P1 is equivalent to

$$
\max _{W_{k}} \sum_{k=1}^{K} R_{k, \mathrm{SD}}^{\mathrm{AF}}
$$

$$
\text { s.t. } \quad \sum_{k=1}^{K} W_{k} \leq W
$$

It can be easily proved that $R_{k, \mathrm{SD}}^{\mathrm{AF}}$ is a concave function of $W_{k}$. Thus, the equivalent problem is convex. Using the Karush-Kuhn-Tucker (KKT) conditions, we can know that the optimal bandwidth allocation $W_{k}^{*}$ must satisfy

$$
\left.\frac{\partial R_{k, \mathrm{SD}}^{\mathrm{AF}}}{\partial W_{k}}\right|_{W_{k}=W_{k}^{*}}=\left.\frac{\partial R_{j, \mathrm{SD}}^{\mathrm{AF}}}{\partial W_{j}}\right|_{W_{j}=W_{j}^{*}}, \quad k, j=1,2, \ldots, K, k \neq j .
$$

Since we have

$$
\begin{aligned}
\left.\frac{\partial R_{k, \mathrm{SD}}^{\mathrm{AF}}}{\partial W_{k}}\right|_{W_{k}=W_{k}^{*}} & \log _{2}\left(1+\frac{P_{k}^{S} P_{k}^{R}\left|h_{k}^{\mathrm{SR}}\right|^{2}\left|h_{k}^{\mathrm{RD}}\right|^{2} \gamma_{0}}{P_{k}^{S}\left|h_{k}^{\mathrm{SR}}\right|^{2} W_{k}^{*}+P_{k}^{R}\left|h_{k}^{\mathrm{RD}}\right|^{2} W_{k}^{*}}\right) \\
& -\frac{P_{k}^{S} P_{k}^{R}\left|h_{k}^{\mathrm{SR}}\right|^{2}\left|h_{k}^{\mathrm{RD}}\right|^{2} \gamma_{0}}{\left(P_{k}^{S} P_{k}^{R}\left|h_{k}^{\mathrm{SR}}\right|^{2}\left|h_{k}^{\mathrm{RD}}\right|^{2} \gamma_{0}+P_{k}^{S}\left|h_{k}^{\mathrm{SR}}\right|^{2} W_{k}^{*}+P_{k}^{R}\left|h_{k}^{\mathrm{RD}}\right|^{2} W_{k}^{*}\right) I n 2} \\
= & Y\left(\frac{P_{k}^{S} P_{k}^{R}\left|h_{k}^{\mathrm{SR}}\right|^{2}\left|h_{k}^{\mathrm{RD}}\right|^{2} \gamma_{0}}{P_{k}^{S}\left|h_{k}^{\mathrm{SR}}\right|^{2} W_{k}^{*}+P_{k}^{R}\left|h_{k}^{\mathrm{RD}}\right|^{2} W_{k}^{*}}\right)
\end{aligned}
$$

where $Y(x) \triangleq \log _{2}(1+x)-\frac{x}{(1+x) \operatorname{In} 2}$. Since $Y(x)$ is a monotonically increasing function for $x>0$, we can obtain from (51) that

$$
\frac{P_{k}^{S} P_{k}^{R}\left|h_{k}^{S \mathrm{SR}}\right|^{2}\left|h_{k}^{\mathrm{RD}}\right|^{2} \gamma_{0}}{P_{k}^{S}\left|h_{k}^{\mathrm{SR}}\right|^{2} W_{k}^{*}+P_{k}^{R}\left|h_{k}^{\mathrm{RD}}\right|^{2} W_{k}^{*}}=\frac{P_{j}^{S} P_{j}^{R}\left|h_{j}^{\mathrm{SR}}\right|^{2}\left|h_{j}^{\mathrm{RD}}\right|^{2} \gamma_{0}}{P_{j}^{S}\left|h_{j}^{\mathrm{SR}}\right|^{2} W_{j}^{*}+P_{j}^{R}\left|h_{j}^{\mathrm{RD}}\right|^{2} W_{j}^{*}} .
$$

It can be easily seen that the optimal bandwidth allocation $W_{k}^{*}$ is obtained when $\sum_{k=1}^{K} W_{k}^{*}=W$. Therefore, using (53), we can obtain the optimal values of $W_{k}^{*}$ given by (11).

\section{Appendix 2}

For the function $f_{k}\left(P_{k}^{S}, P_{k}^{R}\right)=\frac{P_{k}^{S} P_{k}^{R}\left|h_{k}^{\mathrm{SR}}\right|^{2}\left|h_{k}^{\mathrm{RD}}\right|^{2} \gamma_{0}}{P_{k}^{S}\left|h_{k}^{\mathrm{SR}}\right|^{2}+P_{k}^{R}\left|h_{k}^{\mathrm{RD}}\right|^{2}}$, we have

$$
\begin{gathered}
\frac{\partial f_{k}\left(P_{k}^{S}, P_{k}^{R}\right)}{\partial P_{k}^{S}}=\frac{\left(P_{k}^{R}\left|h_{k}^{\mathrm{SR}}\right|\left|h_{k}^{\mathrm{RD}}\right|^{2}\right)^{2} \gamma_{0}}{\left(P_{k}^{S}\left|h_{k}^{\mathrm{SR}}\right|^{2}+P_{k}^{R}\left|h_{k}^{\mathrm{RD}}\right|^{2}\right)^{2}}, \\
\frac{\partial f_{k}\left(P_{k}^{S}, P_{k}^{R}\right)}{\partial P_{k}^{R}}=\frac{\left(P_{k}^{S}\left|h_{k}^{\mathrm{SR}}\right|^{2}\left|h_{k}^{\mathrm{RD}}\right|\right)^{2} \gamma_{0}}{\left(P_{k}^{S}\left|h_{k}^{\mathrm{SR}}\right|^{2}+P_{k}^{R}\left|h_{k}^{\mathrm{RD}}\right|^{2}\right)^{2}}, \\
\frac{\partial^{2} f_{k}\left(P_{k}^{S}, P_{k}^{R}\right)}{\partial\left(P_{k}^{S}\right)^{2}}=-\frac{2\left(P_{k}^{R}\left|h_{k}^{\mathrm{SR}}\right|^{2}\left|h_{k}^{\mathrm{RD}}\right|^{2}\right)^{2} \gamma_{0}}{\left(P_{k}^{S}\left|h_{k}^{\mathrm{SR}}\right|^{2}+P_{k}^{R}\left|h_{k}^{\mathrm{RD}}\right|^{2}\right)^{3}},
\end{gathered}
$$




$$
\begin{aligned}
& \frac{\partial^{2} f_{k}\left(P_{k}^{S}, P_{k}^{R}\right)}{\partial\left(P_{k}^{R}\right)^{2}}=-\frac{2\left(P_{k}^{S}\left|h_{k}^{\mathrm{SR}}\right|^{2}\left|h_{k}^{\mathrm{RD}}\right|^{2}\right)^{2} \gamma_{0}}{\left(P_{k}^{S}\left|h_{k}^{\mathrm{SR}}\right|^{2}+P_{k}^{R}\left|h_{k}^{\mathrm{RD}}\right|^{2}\right)^{3}}, \\
& \frac{\partial^{2} f_{k}\left(P_{k}^{S}, P_{k}^{R}\right)}{\partial P_{k}^{S} \partial P_{k}^{R}}=\frac{\partial^{2} f_{k}\left(P_{k}^{S}, P_{k}^{R}\right)}{\partial P_{k}^{R} \partial P_{k}^{S}}=\frac{2 P_{k}^{S} P_{k}^{R}\left(\left|h_{k}^{\mathrm{SR}}\right|^{2}\left|h_{k}^{\mathrm{RD}}\right|^{2}\right)^{2} \gamma_{0}}{\left(P_{k}^{S}\left|h_{k}^{\mathrm{SR}}\right|^{2}+P_{k}^{R}\left|h_{k}^{\mathrm{RD}}\right|^{2}\right)^{3}} .
\end{aligned}
$$

Thus, from (54)-(58), it follows that

$$
\begin{aligned}
& \frac{\partial^{2} f_{k}\left(P_{k}^{S}, P_{k}^{R}\right)}{\partial\left(P_{k}^{S}\right)^{2}} \leq 0 \\
& \frac{\partial^{2} f_{k}\left(P_{k}^{S}, P_{k}^{R}\right)}{\partial\left(P_{k}^{R}\right)^{2}} \leq 0 \\
& \left(\frac{\partial^{2} f_{k}\left(P_{k}^{S}, P_{k}^{R}\right)}{\partial\left(P_{k}^{S}\right)^{2}}\right)\left(\frac{\partial^{2} f_{k}\left(P_{k}^{S}, P_{k}^{R}\right)}{\partial\left(P_{k}^{R}\right)^{2}}\right)-\left(\frac{\partial^{2} f_{k}\left(P_{k}^{S}, P_{k}^{R}\right)}{\partial P_{k}^{S} \partial P_{k}^{R}}\right) \\
& \left(\frac{\partial^{2} f_{k}\left(P_{k}^{S}, P_{k}^{R}\right)}{\partial P_{k}^{R} \partial P_{k}^{S}}\right)=0 .
\end{aligned}
$$

Since the Hessian matrix of $f_{k}\left(P_{k}^{S}, P_{k}^{R}\right)$ is given by

$$
\left[\begin{array}{c}
\frac{\partial^{2} f_{k}\left(P_{k}^{S}, P_{k}^{R}\right)}{\partial\left(P_{k}^{S}\right)^{2}} \frac{\partial^{2} f_{k}\left(P_{k}^{S}, P_{k}^{R}\right)}{\partial P_{k}^{S} \partial P_{k}^{R}} \\
\frac{\partial^{2} f_{k}\left(P_{k}^{S}, P_{k}^{R}\right)}{\partial P_{k}^{R} \partial P_{k}^{S}} \frac{\partial^{2} f_{k}\left(P_{k}^{S}, P_{k}^{R}\right)}{\partial\left(P_{k}^{R}\right)^{2}}
\end{array}\right],
$$

the Hessian matrix of $f_{k}\left(P_{k}^{S}, P_{k}^{R}\right)$ is a negative semi-definite matrix. As a consequence, it is implied that $f_{k}\left(P_{k}^{S}, P_{k}^{R}\right)$ is concave.

Since the objective function of Problem P3 is $\sum_{k=1}^{K} f_{k}\left(P_{k}^{S}, P_{k}^{R}\right)$, it follows that the objective function of Problem P3 is concave in $P_{k}^{S}$ and $P_{k}^{R}(k=1,2, \ldots, K)$.

\section{Competing interests}

The authors declare that they have no competing interests.

\section{Acknowledgements}

This study was supported in part by National Natural Science Foundation of China under Grant No. 61271169, National Basic Research Program $(973$ Program) of China under Grant No. 2009CB320405, Nation Grand Special Science and Technology Project of China under Grant Nos. 2010ZX03006-002, 2010ZX03002-008-03.

\section{Received: 23 December 2011 Accepted: 20 September 2012}

Published: 22 October 2012

\section{References}

1. J Mitola, GQ Maguire, Cognitive radio: making software radios more personal, IEEE Pers. Commun. 6(4), 13-18 (1999)

2. S Haykin, Cognitive radio: brain-empowered wireless communications, IEEE J. Sel. Areas Commun. 23(2), 201-220 (2005)

3. BM Q Zhao, A Sadler, survey of dynamic spectrum access, IEEE Signal Process. Mag. 24(3), 79-89 (2007)

4. Q Zhao, A Swami, A decision-theoretic framework for opportunistic spectrum access, IEEE Wirel. Commun. Mag. 14(4), 14-20 (2007)
5. A Ghasemi, ES Sousa, Fundamental limits of spectrum-sharing in fading environments, IEEE Trans. Wirel. Commun. 6(2), 649-658 (2007)

6. X Kang, Y-C Liang, A Nallanathan, H Krishna, R Zhang, Optimal power qllocation for fading channels in cognitive radio networks: ergodic capacity and outage capacity, IEEE Trans. Wirel. Commun. 8(2), 940-950 (2009)

7. X Gong, SA Vorobyov, C Tellambura, Optimal bandwidth and power allocation for sum ergodic capacity under fading channels in cognitive radio networks, IEEE Trans. Signal Process. 59(4), 1814-1826 (2011)

8. V Asghari, S Aissa, Resource management in spectrum-sharing cognitive radio broadcast channels: adaptive time and power allocation, IEEE Trans. Wirel. Commun. 59(5), 1446-1457 (2011)

9. SM Almalfouh, GL Stüber, Interference-aware radio resource allocation in OFDMA-based cognitive radio networks, IEEE Trans. Veh. Technol. 60(4), 1699-1713 (2011)

10. L Li, X Zhou, H Xu, GY Li, D Wang, A Soong, Simplified relay selection power allocation in cooperative cognitive radio systems, IEEE Trans. Wirel. Commun. 10(1), 33-36 (2011)

11. G Zhao, C Yang, GY Li, D Li, ACK Soong, Power and channel allocation for cooperative relay in cognitive radio networks, IEEE J. Sel. Topics Signal Process. 5(1), 151-159 (2011)

12. D Bharadia, G Bansal, P Kaligineedi, VK Bhargava, Relay and power allocation schemes for OFDM-based cognitive radio systems, IEEE Trans. Wirel. Commun. 10(9), 2812-2817 (2011)

13. $L \mathrm{Li}, X \mathrm{Zhou}, \mathrm{H} X \mathrm{Xu}, \mathrm{GY} \mathrm{Li}, \mathrm{D}$ Wang, A Soong. Energy-efficient transmission in cognitive radio networks, in Proc. IEEE Consumer Communications and Network Conference (CCNC), (Las Vegas, NV , USA, 2010, pp. 1-5

14. Y Pei, Y-C Liang, KC Teh, KH Li, Energy-efficient design of sequential channel sensing in cognitive radio networks: optimal sensing strategy, power allocation, and sensing order, IEEE J. Sel. Areas Commun. 29(8), 1648-1659 (2011)

15. M Sniedovich, Dynamic Programming. (Marcel Dekker, New York, 2011)

16. JM Peha, Approaches to spectrum sharing, IEEE Commun. Mag. 43(2), 10-12 (2005)

17. N Krishnan, RD Yates, NB Mandayam, JS Panchal, Bandwidth sharing for relaying in cellular systems, IEEE Trans. Wirel. Commun. 11(1), 117-129 (2012)

18. K Kumaran, $\mathrm{H}$ Viswanathan, Joint power bandwidth allocation in downlink transmission, IEEE Trans. Wirel. Commun. 4(3), 1008-1016 (2005)

19. Y Zhao, R Adve, TJ Lim, Improving amplify-and-forward relay networks: optimal power allocation versus selection, IEEE Trans. Wirel. Commun. 6(8), 3114-3123 (2007)

20. X Gong, SA Vorobyov, C Tellambura, Joint bandwidth and power allocation with admission control in wireless multi-user networks with and without relaying, IEEE Trans. Signal Process. 59(4), 1801-1813 (2011)

21. S Sadr, A Anpalagan, K Raahemifar, Radio resource allocation algorithms for the downlink of multiuser OFDM communication systems, IEEE Commun. Surv. Tutor. 11(3), 92-106 (2009)

doi:10.1186/1687-6180-2012-224

Cite this article as: Yu et al:: Bandwidth and power allocation for cooperative relay in cognitive radio networks. EURASIP Journal on Advances in Signal Processing 2012 2012:224. 